\title{
ON FINITE PRESENTATIONS FOR GROUPS
}

\author{
R. CRAGGS
}

\begin{abstract}
Three theorems are proved here giving canonical forms for finitely-presented groups. The first characterizes groups with balanced presentations as those groups with presentations of the form
\end{abstract}

$$
\left\langle y_{1}, \ldots, y_{n} \mid y_{i}=\alpha\left(y_{i}\right), i=1, \ldots, n\right\rangle
$$

where $\alpha$ is some automorphism of the free group with free basis $y_{1}, \ldots, y_{n}$. Similar canonical forms, with two automorphisms rather than one, are given for arbitrary finitely-presented groups.

For each $n \geqslant 1$, let $Y^{n}$ denote the free group on the alphabet $y_{1}, \ldots, y_{n}$, and consider finite group presentations of the form

$$
\mathscr{P}=\left\langle y_{1}, \ldots, y_{n} \mid r_{1}, \ldots, r_{p}\right\rangle \text {. }
$$

Here each relator $r_{i}$ is an element of $Y^{n}$, and $\mathcal{P}$ is a presentation for a group $H$ if there is an epimorphism $\eta: Y^{n} \rightarrow H$ whose kernel $N$ is the smallest normal subgroup of $Y^{n}$ containing each of the relators $r_{i}$. For automorphisms $\alpha_{1}, \ldots, \alpha_{k}$ of $Y^{n}$, consider also finite presentations with $n$ generators and $k \cdot n$ relators,

$$
\mathscr{P}\left(\alpha_{1}, \ldots, \alpha_{k}\right)=\left\langle y_{1}, \ldots, y_{n} \mid y_{j}^{-1} \alpha_{i}\left(y_{j}\right), 1 \leqslant i \leqslant k, 1 \leqslant j \leqslant n\right\rangle .
$$

Below we prove three theorems showing how to convert any finite presentation for a group to one of the canonical forms $\mathscr{P}\left(\alpha_{1}, \ldots, \alpha_{k}\right)$. In particular, we characterize groups with balanced presentations by showing that such presentations can be converted to the form above with $k=1$. These forms are suggested by the theory of HNN-extensions and will be discussed briefly after the theorems are proved. In the proofs of the theorems we will make use of Nielsen and Schreier's theorem that any set of $n$ elements which generates a free group of rank $n$ freely generates that group (see Magnus, Karass, and Solitar [12, §3.2] or Lyndon and Schupp [11, Chapter 1, §2]).

THEOREM A. Let $H$ be any finitely-presented group with balanced presentation (no. generators $=$ no. relators)

$$
\mathscr{P}=\left\langle y_{1}, \ldots, y_{n} \mid r_{1}, \ldots, r_{n}\right\rangle .
$$

Then for some automorphism $\alpha$ of $Y^{2 n}$, the group $H$ has a presentation $\mathcal{P}(\alpha)$.

PrOof. Our proof is suggested by a construction in Rapaport [22, p. 230]. Consider the homomorphism $\alpha$ of $Y^{2 n}$ defined by

$$
\alpha\left(y_{i}\right)=r_{i} y_{i+n}, \quad \alpha\left(y_{i+n}\right)=y_{i}, \quad i \leqslant n .
$$

Received by the editors December 8, 1978.

AMS (MOS) subject classifications (1970). Primary 20F05, 20E36; Secondary 20E06, 57M05. 
This is certainly an automorphism since $\left\{r_{i} y_{i+n}\right\} \cup\left\{y_{i}\right\}$ generates $Y^{2 n}$ and so freely generates $Y^{2 n}$. But the presentation $\mathscr{P}(\alpha)$ results from $\mathscr{P}$ by the following sequence of operations.

(1) Add new generators $y_{n+1}, \ldots, y_{2 n}$ and new relators $y_{i+n}^{-1} y_{i}$.

(2) Conjugate each old relator $r_{i}$ to $y_{i}^{-1} r_{i} y_{i}$.

(3) Multiply each modified relator $\left(y_{i}^{-1} r_{i} y_{i}\right)$ on the right by $\left(y_{i+n}^{-1} y_{i}\right)^{-1}$.

Each of these operations is a Tietze transformation; so $\mathscr{P}(\alpha)$ must also be a presentation for $H$.

THEOREM B. Let $\boldsymbol{H}$ be any finitely-presented group. For some $n$ and for some pair of automorphisms $\alpha$ and $\beta$ of $Y^{n}$, the group $H$ has a presentation $\mathscr{P}(\alpha, \beta)$.

Proof. Let $H$ have the presentation

$$
\mathscr{P}=\left\langle y_{1}, \ldots, y_{m} \mid r_{1}, \ldots, r_{p}\right\rangle .
$$

By adding, if necessary, new generators $y_{i}(i>m)$ and adding the same $y_{i}$ 's as relators, we may arrange things so that $p<2 \mathrm{~m}$. Then by adding copies of the trivial relator 1 , we may arrange things so that $p=2 m$. Set $n=2 m$ and $s_{i}=r_{i+m}$ $(i \leqslant m)$. Define, as in the proof of Theorem A, two automorphisms $\alpha$ and $\beta$ by

$$
\begin{aligned}
\alpha\left(y_{i}\right) & =r_{i} y_{i+m} ; \quad \beta\left(y_{i}\right)=s_{i} y_{i+m}, \\
\alpha\left(y_{i+m}\right) & =y_{i} ; \quad \beta\left(y_{i+m}\right)=y_{i} .
\end{aligned}
$$

Now, just as in the proof of Theorem $\mathrm{A}$, the group $H$ has the presentation $\mathscr{P}(\alpha, \beta)$.

The deficiency of a presentation $\mathscr{P}=\left\langle y_{1}, \ldots, y_{n} \mid r_{1}, \ldots, r_{p}\right\rangle$ is the difference $p-n$ between the number of relators and the number of generators. The deficiency of a finitely presented group $H$ is the minimum deficiency taken over all finite presentations for $H$. For a finitely-presented group $H$, let $k(H)$ denote the maximum of 2 and the deficiency of $H$. Finally, for each pair of natural numbers $(k, n)$ with $2 \leqslant k \leqslant n$, let $\beta_{k, n}$ denote the automorphism of $Y^{n}$ defined by

$$
\begin{gathered}
\beta_{k, n}\left(y_{i}\right)=y_{i+1} \quad(i<k), \quad \beta_{k, n}\left(y_{i}\right)=y_{i} \quad(i>k), \\
\beta_{k, n}\left(y_{k}\right)=y_{k} y_{1} .
\end{gathered}
$$

The next theorem shows that $\mathscr{P}(\alpha, \beta)$ in Theorem B can always be chosen so that $\beta=\beta_{k, n}$ for some pair $(k, n)$.

ThEOREM C. Let $H$ be any finitely-presented group and let $k=k(H)$. For some $n$, the group $H$ has a presentation $\mathcal{P}\left(\alpha, \beta_{k, n}\right)$ as in Theorem $\mathrm{B}$.

Proof. Let $H$ have a presentation

$$
\mathcal{P}=\left\langle y_{1}, \ldots, y_{m} \mid r_{1}, \ldots, r_{p}\right\rangle
$$

with minimum deficiency. If this deficiency is smaller than 2, add copies of the trivial relator to $\mathscr{P}$ so that $p-m=k \geqslant 2$. Now

$$
\left\langle y_{1}, \ldots, y_{m}, \ldots, y_{p} \mid r_{1}, \ldots, r_{p}\right\rangle
$$

is a balanced presentation for the group $W * H$ where $W$ is a free group of rank $k=p-m$. 
By Theorem $A$ there is a natural number $n$ and there is an automorphism $\alpha_{1}$ of $Y^{n}$ so that $W * H$ has the presentation $\mathscr{P}\left(\alpha_{1}\right)$. This presentation corresponds to an epimorphism $\eta: Y^{n} \rightarrow W * H$ whose kernel is normally generated by the relator set $\left\{y_{i}^{-1} \alpha_{1}\left(y_{i}\right)\right\}$. By the Grushko-Neumann theorem (see Grushko [6], Neumann [18], Kurosh [9], Lyndon [10], Lyndon and Schupp [11], Massey [14], or Stallings [24]), $Y^{n}$ decomposes as a free product of free groups $U * V$ where $\eta$ maps $U$ onto $W$. By Lyndon and Schupp [11, Chapter 1,2.12] we may suppose that $\eta$ maps $U$ isomorphically onto $W$. By the Nielsen-Schreier theorem on free generators, there is an automorphism $\gamma$ of $Y^{n}$ taking $Y^{k}$ onto $U$. This automorphism converts $\mathscr{P}\left(\alpha_{1}\right)$ to the presentation

$$
\left\langle y_{1}, \ldots, y_{n} \mid \gamma^{-1}\left(y_{i}\right)=\gamma^{-1} \alpha_{1}\left(y_{i}\right)(i \leqslant n)\right\rangle
$$

corresponding to the epimorphism $\eta \circ \gamma$ (order of composition $y_{i} \rightarrow \eta\left(\gamma\left(y_{i}\right)\right.$ )). But we can use any generating set in place of $\left\{y_{i}\right\}$ to write an equivalent set of relations. Thus

$$
\left\langle y_{1}, \ldots, y_{n} \mid \gamma^{-1}\left(\gamma\left(y_{i}\right)\right)=\gamma^{-1} \alpha_{1} \gamma\left(y_{i}\right)\right\rangle
$$

is a presentation for $W * H$. But if $\alpha=\gamma^{-1} \alpha_{1} \gamma$, then the presentation above is just $\mathscr{P}(\alpha)$ with the relators rewritten as relations. Now $\mathscr{P}(\alpha)$ corresponds to the epimorphism $\eta \circ \gamma$. Since $\eta \circ \gamma$ carries $Y^{k}$ isomorphically onto $W$, we need only kill $y_{1}, \ldots, y_{k}$ to get a presentation for $H$. The relator set $\left\{y_{i}^{-1} \beta_{k, n}\left(y_{i}\right)\right\}$ generates the subgroup $Y^{k}$ and so $\mathscr{P}\left(\alpha, \beta_{k, n}\right)$ is the desired presentation for $H$.

REMARKS. I. It should be noted that the presentation $\mathscr{P}(\alpha)$ in Theorem $A$ is obtained from $\mathcal{P}$ by the application of extended Nielsen transformations (see Andrews and Curtis [2], [3], Metzler [15], Pfeiffer [20], Rapaport [23], and Wright [25] for a discussion of extended Nielsen transformations).

II. The presentations $\mathscr{P}(\alpha)$ and $\mathscr{P}(\alpha, \beta)$ are closely related to presentations for HNN-extensions of free groups $\overline{\mathcal{P}}(\alpha)$ and $\overline{\mathcal{P}}(\alpha, \beta)$ described below.

$$
\begin{aligned}
\overline{\mathcal{P}}(\alpha) & =\left\langle y_{1}, \ldots, y_{2 n}, t \mid t^{-1} y_{i} t=\alpha\left(y_{i}\right)(1 \leqslant i \leqslant 2 n)\right\rangle, \\
\overline{\mathcal{P}}(\alpha, \beta) & =\left\langle y_{1}, \ldots, y_{n}, s, t \mid s^{-1} y_{i} s=\alpha\left(y_{i}\right), t^{-} y_{i} t=\beta\left(y_{i}\right)(1 \leqslant i \leqslant n)\right\rangle .
\end{aligned}
$$

In each case we obtain the canonical presentation $\mathscr{P}(\alpha)$ or $\mathscr{P}(\alpha \beta)$ by killing the stable letter or letters ( $t$ or $s$ and $t$ ) in the displayed presentation. (For further discussion of HNN-extensions see Higman, Neumann and Neumann [8], Miller [16], or Lyndon and Schupp [11, Chapter 4, §7].)

III. The presentations $\overline{\mathcal{P}}(\alpha)$ described in II arise naturally in 3-manifold topology as presentations for fundamental groups of fibered knots. We discuss this connection briefly, for it offers some insight into the special properties of some groups with balanced presentations that make them 3-manifold groups.

Every closed, compact, oriented 3-manifold $M$ contains a fibered knot (Myers [17], González-Acuña [5]). The closure of the complement of such a knot fibers over the circle. Thus there is a compact, oriented surface $Q$, of genus say $n$ and with one boundary component, so that the fibered space just mentioned has a description as $Q \times[0,1] /(y, 0)=(h(y), 1)$ where $h$ is some orientation-preserving homeomorphism of $Q$ that leaves $\mathrm{Bd} Q$ pointwise fixed. The fundamental group of 
$Q$ is free on $2 n$ generators, say $y_{1}, \ldots, y_{2 n}$, and the fundamental group of the knot complement has a presentation as $\overline{\mathcal{P}}\left(h_{*}\right)$ (see for example Lyndon and Schupp [11, p. 190]). Here $t$ corresponds to the class of a meridian on the boundary of the tubular neighborhood of the knot, and $h_{*}$ is the automorphism of $\pi_{1}(Q)$ induced by $h$. A corresponding presentation for the fundamental group $\pi_{1}(M)$ is then $\mathscr{P}\left(h_{*}\right)$. It is obtained from $\overline{\mathcal{P}}\left(h_{*}\right)$ by killing $t$.

What makes $\overline{\mathscr{P}}\left(h_{*}\right)$ and $\mathscr{P}\left(h_{*}\right)$ more special than the average $\overline{\mathcal{P}}(\alpha)$ and $\mathscr{P}(\alpha)$ is this: If the base point for $\pi_{1}(Q)$ is chosen on $\mathrm{Bd} Q$, and if the basis $y_{1}, \ldots, y_{2 n}$ is chosen appropriately, then $h_{*}(q)=q$ where $q=\Pi_{1}^{n}\left[y_{i}, y_{i+n}\right]$. Nielsen's technique for geometrically realizing automorphisms of surface groups (see Nielsen [19] and Mangler [13]) shows that the reverse is true. If an automorphism $\alpha$ of $Y^{2 n}$ leaves the quadratic word $q$ fixed, then $\alpha$ is induced by a homeomorpnism of a compact oriented surface $Q$ with one boundary component. In that case $\overline{\mathcal{P}}(\alpha)$ is a presentation for the group of a fibered knot complement in a 3-manifold $M$ and $\mathscr{P}(\alpha)$ is a presentation for $\pi_{1}(M)$.

Thus we see that to effectively determine whether a group with balanced presentation is the group of a closed, oriented 3-manifold, one must be able to determine whether there is a presentation $\mathscr{P}(\alpha)$ for the group, based on $\alpha$ : $Y^{2 n} \rightarrow Y^{2 n}$, such that $\alpha$ leaves the quadratic word $q$ fixed.

IV. The triviality problem for groups with balanced presentations-it seeks an algorithm that determines, for each balanced presentation of a group, whether the group is trivial-is a major unsolved problem in combinatorial group theory. The triviality problem for arbitrary finitely-presented groups is, nevertheless, known to be unsolvable (see Adjan [1], Rabin [21], also Boone [4] and Miller [16, Chapter V, Theorem 1]). One possible approach to this problem is developed below. It is based on a notion of embedding presentations, and for the sake of simplicity we restrict things to the case of balanced presentations.

Let $\mathscr{P}(\alpha)$ and $\mathscr{P}(\beta)$ be presentations based on automorphisms $\alpha$ of $Y^{n}$ and $\beta$ of $Y^{m}$. We say that $\mathscr{P}(\alpha)$ embeds in $\mathscr{P}(\beta)$ if there is a subgroup $W$ of $Y^{m}$ isomorphic to $Y^{n}$ under an isomorphism $\gamma: Y^{n} \rightarrow W$ so that

(1) $W$ is invariant under $\beta$,

(2) $\alpha=\gamma^{-1} \circ \beta \circ \gamma$ and

(3) $\gamma$ induces a monomorphism of the factor group $Y^{n} / \alpha$ into the factor group $Y^{m} / \beta$.

Notice that the definition make sense when we allow $m, n$, or both to be infinite. Furthermore, Theorems A, B, and C have analogs, with identical proofs, in the case of free groups with infinite rank. When an infinite number of generators is allowed, every recursively-presented group has a balanced presentation with an infinite number of generators and an infinite number of relators. It follows that every recursively-presented group has a presentation as $Y^{\omega} / \alpha$ where $Y^{\omega}$ is a free group of infinite rank and $\alpha$ is some recursively defined automorphism on $Y^{\omega}$.

Consider the following three questions on embeddings of presentations. The first two are suggested by the Higman embedding theorem [7].

(1) If $\mathscr{P}(\alpha)$ is a presentation based on $\alpha: Y^{\omega} \rightarrow Y^{\omega}$, does $\mathscr{P}(\alpha)$ embed, in some effective manner, in some finite presentation $\mathscr{P}(\beta)$ ? 
(2) If the answer to (1) is "no", can $\alpha$ be replaced, in some effective way, by a new automorphism $\alpha^{\prime}$ so that $\mathscr{P}\left(\alpha^{\prime}\right)$ presents the same group and embeds in some finite presentation $\mathscr{P}(\beta)$ ?

(3) If the answer to (1) or (2) is "yes", can $\beta$ be chosen so that $\mathscr{P}(\beta)$ presents the trivial group if $\mathscr{P}(\alpha)$ does?

If the answer to (3) were "yes", and if one could effectively determine when one had embeddings, then it would follow easily that the triviality problem would be unsolvable for groups with finite balanced presentations.

\section{REFERENCES}

1. S. I. Adjan, On algorithmic problems in effectively complete classes of groups, Dokl. Akad. Nauk SSSR 123 (1958), 13-16.

2. J. J. Andrews and M. L. Curtis, Free groups and handlebodies, Proc. Amer. Math. Soc. 16 (1965), 192-195.

3. Extended Nielsen operations in free groups, Amer. Math. Monthly 73 (1960), 21-28.

4. W. W. Boone, Decision problems about algebraic and logical systems as a whole and recursively enumerable degrees of unsolvability, Contributions to Mathematical Logic, K. Schütte, editor, North-Holland, Amsterdam, 1968.

5. F. González-Acuña, 3-dimensional open books, Lectures, Univ. Iowa Topology Seminar, 1974/1975.

6. I. A. Grushko, Über die Basen eines freien Produktes von Gruppen, Mat. Sb. 8 (1940), 160-182.

7. G. Higman, Subgroups of finitely presented groups, Proc. Roy. Soc. London Ser. A 262 (1961), $455-475$.

8. G. Higman, B. H. Neumann and H. Neumann, Embedding theorems for groups, J. London Math Soc. 24 (1949), 247-254.

9. A. G. Kurosh, The theory of groups. Vol. II, 2nd ed., translated from the Russian and edited by K. A. Hirsch, Chelsea, New York, 1960.

10. R. C. Lyndon, Grushko's theorem, Proc. Amer. Math. Soc. 16 (1965), 822-826.

11. R. C. Lyndon and P. E. Schupp, Combinatorial group theory, Ergebnisse der Math. und ihrer Grenzgebiete, neue folge, Bd. 89, Springer-Verlag, Berlin and New York, 1976.

12. W. Magnus, A. Karass and D. Solitar, Combinatorial group theory: Presentations of groups in terms of generators and relations, Pure and Appl. Math., vol. 12, Interscience [Wiley], New York, 1966.

13. W. Mangler, Die Klassen von topologischen Abbildungen einer geschlossenen Fläche auf sich, Math. Z. 44 (1938), 541-544.

14. W. S. Massey, Algebraic topology: An introduction, Harcourt, Brace and World, New York, 1967.

15. W. Metzler, Uber den Homotopietyp zweidimensionaler $C W$-Komplexe und Elementartransformationen bei Darstellungen von Gruppen durch Erzeugende und definierende Relationen, J. Reine Angew. Math. 235 (1976), 7-23.

16. C. F. Miller III, On group-theoretic decision problems and their classification, Ann. of Math. Studies, no. 68, Princeton Univ. Press, Princeton, N.J.; Univ. of Tokyo Press, Tokyo, 1971.

17. R. Myers, Open book decompositions of 3-manifolds, Proc. Amer. Math. Soc. 72 (1978), 397-402.

18. B. H. Neumann, On the number of generators in a free product, J. London Math. Soc. 18 (1943), 12-20.

19. J. Nielsen, Untersuchungen zur Topologie der geschlossenen zweiseitigen Flächen, Acta Math. 50 (1927), 189-358.

20. R. Pfeiffer, Über Identitäten zwischen Relationen, Math. Ann. 121 (1949), 67-99.

21. M. O. Rabin, Recursive unsolvability in group theoretic problems, Ann. of Math. (2) 67 (1958), 172-194.

22. E. S. (Elvira Strasser) Rapaport, Note on Nielsen transformations, Proc. Amer. Math. Soc. 10 (1959), 228-235.

23. __ Groups of order 1: Some properties of presentations, Acta Math. 121 (1968), 127-150.

24. J. Stallings, A topological proof of Grushko's theorem on free products, Math. Z. 90 (1965), 1-8.

25. P. Wright, Group presentations and formal deformations, Trans. Amer. Math. Soc. 208 (1975), $161-169$.

Department of Mathematics, University of Illinois at Urbana-Champaign, Urbana, Illinois 61801 